\title{
„Wird erst einmal der Macer gedruckt sein....
}

\author{
Die Pflanzen des mittelalterlichen Lehrgedichtes „Macer floridus“ und ihre \\ heutige Bedeutung
}

Tobias Niedenthal

Forschergruppe Klostermedizin, Würzburg

\section{ZUSAMMENFASSUNG}

Der „Macer floridus“ (alternativ „De viribus herbarum“) gehört zweifellos zu den wichtigsten Werken der europäischen Pflanzenheilkunde. Das lateinische Lehrgedicht aus dem 11. Jahrhundert, das in 77 Kapiteln die wichtigsten Arzneipflanzen der Zeit behandelt, ist der Allgemeinheit heute eher unbekannt, was nicht zuletzt daran liegen mag, dass es bereits relativ kurz nach seiner Entstehung in nahezu alle wichtigen europäischen Volkssprachen übertragen und noch im Mittelalter vielfach ungenannt in Kräuterbuch-Kompilationen eingearbeitet wurde. Während die Bedeutung des Werkes in seiner Gänze in der Neuzeit zunehmend verblasste, wurden zahlreiche Anwendungen noch über Jahrhunderte tradiert.
Zur deutschen Vulgatfassung, die auf die Zeit um 1220 datiert wird, schrieb der Wiener Bibliothekar Josef Haupt 1872:

„Wird erst einmal der Macer gedruckt sein, wird auch blöden Augen klar sein, dass eine Masse nicht nur unserer Hausmittel auf gelehrtem Wege unters Volk gekommen ist, sondern dass daher und aus dem Apuleius das meiste des Aberglaubens von den Pflanzen stammt, womit man in der ,deutschen Mythologie‘ so fürchterlich Staat gemacht hat. " [4]

Haupt kritisiert hier deutlich das Vorgehen Jacob Grimms in seinem erstmals 1835 vorgelegten Werk zur Mythologie der Germanen. Der ebenfalls erwähnte, spätantike „Herbarius“ des (Pseudo-)Apuleius war bis ins Hochmittelalter ein bestimmendes Kräuterbuch des lateinischen Mittelalters, das meist in Verbund mit weiteren Traktaten zu pflanzlichen und animalischen Drogen überliefert wurde.

Zum Autoren des „Macer floridus“ wissen wir wenig mehr als seinen Namen: Odo Magdunensis (Odo von Meung). Als gesichert kann gelten, dass Odo einen klerikalen Hintergrund hatte, zudem Zugriff auf eine gut ausgestattete Bibliothek. Ob er selbst als Arzt tätig war, ist hingegen unklar [15], S.27. Zugeschrieben wurde das Werk bereits früh dem antiken Autoren Aemilius Macer aus dem 1. Jahrhundert, dessen verschollenes Kräutergedicht zumindest als Vorlage gedient haben soll. Dies muss aus heutiger Sicht verneint werden, der Titel „Macer floridus“ ist aber weiterhin geläufig. Zu
Hintergrund und aktuellem Forschungsstand hinsichtlich Werk und Autor sei an dieser Stelle auf den einführenden Beitrag von Johannes G. Mayer und Konrad Goehl in dieser Zeitschrift verwiesen [10] und darüber hinaus auf die entsprechenden Abschnitte in den Editionen der deutschen Vulgatfassung [15] ( A Abb. 1) und der als „Spuria Macri“ bezeichneten Ergänzungskapitel [7] (॰ Abb. 1).

Neben seiner großen Bedeutung für die europäische Pflanzenheilkunde, die teils noch heute in „Hagers Enzyklopädie“ unter „volkstümliche Anwendungen“ anklingt [12], S.35, bietet sich der „Macer“ noch aus weiteren Gründen für eine nähere Betrachtung an. Obwohl das Werk mittlerweile fast 950 Jahre alt ist, sind fast alle erwähnten Pflanzen heute relativ eng einzugrenzen, denn die damals verwendeten Namen sind oftmals Bestandteil der modernen botanischen Nomenklatur geworden. Für diesen Beitrag konnte daher im Wesentlichen auf die bisherigen Auflagen der neuhochdeutschen Übersetzung zurückgegriffen werden [9], [11], [12]. In wenigen Fällen wurde die Identifikation noch einmal kritisch hinterfragt, wobei der deutschen Vulgatfassung eine besondere Rolle zuteil wurde. Hier ergaben sich einige interessante Widersprüche. Wenig hilfreich bei der Identifikation ist hingegen der illustrierte Frühdruck des lateinischen Textes (mehrere Ausgaben um 1500) [2], da die Holzschnitte sehr stark stilisiert sind.

Der Umfang von 77 Pflanzenkapiteln, in denen bisweilen mehrere Pflanzen erwähnt werden, kann zudem als repräsentativ für die Zeit gelten. Gegenüber seinem Vorläufer, dem „Hortulus“ von Walahfrid Strabo aus der 


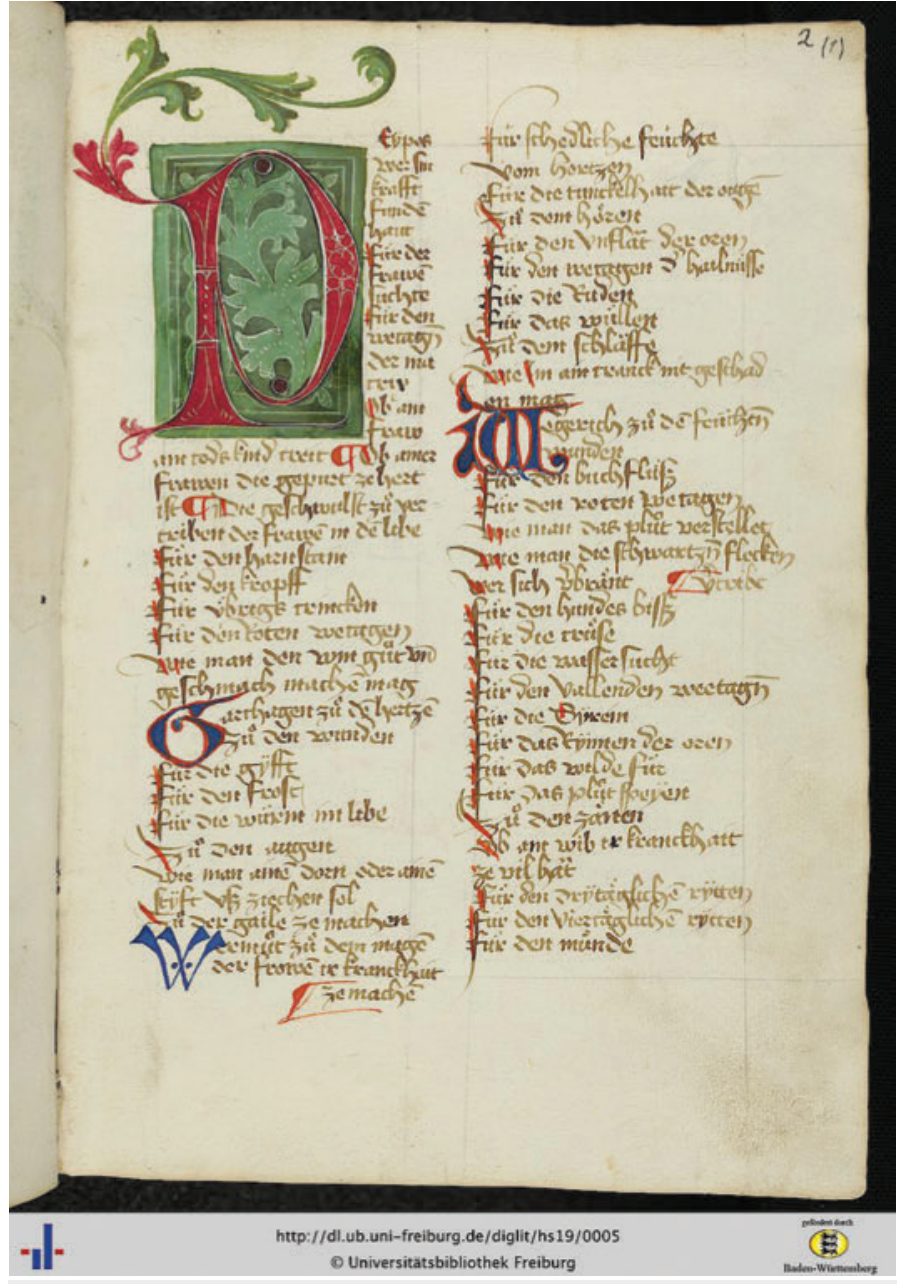

-Abb. 1 „Schöne“ Handschriften des „Macers“ sind rar, in der Universitätsbibliothek Freiburg gibt es diesen Textzeugen in einer deutsch-lateinischen medizinischen Sammelhandschrift (Memmingen, 1463 -1480). Es handelt sich hier um eine Art Übersicht vor der eigentlichen Abschrift der deutschen Vulgatfassung. Quelle: Universitätsbibliothek Freiburg i.Br./Historische Sammlungen. Hs. 19. http://dl.ub.uni-freiburg.de/diglit/hs19/0005

ersten Hälfte des 9. Jahrhunderts, in dem lediglich 24 Pflanzen in 23 Kapiteln beschrieben werden, bietet der „Macer“ mehr als dreimal so viele Pflanzen. Gegenüber anderen Kräuterbüchern konzentriert sich Odo jedoch auf die wichtigsten. Der bereits erwähnte „Herbarius“ (Pseudo-Apuleius) aus der Spätantike enthält gekoppelt mit „De herbis femininis“ (Pseudo-Dioskurides) insgesamt rund 200 Pflanzenkapitel (bei einigen Überschneidungen), die vor allem aus Plinius und Dioskurides schöpfen. Der Erfolg dieses Korpus im frühen und hohen Mittelalter erklärt sich in erster Linie durch die stilisierten Abbildungen, die ihren Höhepunkt im 13. Jahrhundert im Umfeld von Kaiser Friedrich II. erreichten (unter Vernachlässigung des Textes). Ähnlich umfangreich hinsichtlich der Zahl der beschriebenen Pflanzen sind das „Circa instans“ aus Salerno sowie die Naturkunde Hildegards von Bingen, beide aus der Mitte des 12 .
Jahrhunderts. Das „Circa instans“ wurde im Spätmittelalter bis auf weit über 400 Kapitel erweitert, während die Naturkunde Hildegards nur vergleichsweise spärlich rezipiert wurde. Selbst der „Liber graduum“ des Constantinus Africanus, Vorlage für die exotischen Gewürze im „Macer“, kommt auf über 200 Pflanzen.

\section{Methodik}

Für alle identifizierten Pflanzen wurde überprüft, ob Monografien der Kommission E, der ESCOP oder des HMPC vorliegen, wobei für die Kommission E negative Monografien und sog. „Nullmonografien“ gesondert erfasst wurden. Auf diese Weise kann punktuell die Entwicklung der Phytotherapie in den letzten Jahrzehnten nachvollzogen werden. Insbesondere für die Pflanzen ohne positive Monografie wurde zudem erfasst, ob sie noch im DAB 6 bzw. EB 6 (Neudruck 1947 / 48, gültig bis 1968) erwähnt werden und ob sie weiterhin als Lebensmittel (Gemüse, Gewürz, Tee...) oder in der Kosmetik Verwendung finden ( $\triangleright$ Tab. 1).

\section{Strittige Kapitel und Besonderheiten}

Vor allem das 50. Kapitel zu 'Barrocum' (in der deutschen Vulgatfassung als Kapitel 73 unter 'Baricum' und 'Barotum') wirft bis heute Fragen auf. Gemeinhin wird hier seit dem 19. Jahrhundert die Melisse vermutet, alternativ das kaum verwendete Immenblatt. Auf beide deutet der im "Macer“ genannte griechisch-lateinische Name 'melissophyllum' bzw. 'melissophilon'. Ebenso gut denkbar ist allerdings die Weiße Taubnessel, und Hermann Fischer nannte 1929 mit dem Mädesüß auch noch eine Möglichkeit außerhalb der Familie der Lippenblütler [3]. Im „Gart der Gesundheit“ (1485) findet sich in Kapitel 69 unter den Namen 'Barotus' und 'Bynßauge' die Weiße Taubnessel, die Melisse im Kapitel 250 unter 'Melissa' und 'Muterkrut'. Gerade der deutsche Trivialname Bienensaug, der regional auch heute noch für Taubnesseln verwendet wird (etwa Roter Bienensaug für die Purpurrote Taubnessel, Lamium purpureum), gemahnt hier zur Vorsicht, denn alle genannten Lippenblütler tragen somit die Biene im Namen. Dies gilt analog auch für 'binesuge' in Hildegards Naturkunde (I.59), was ebenfalls nicht zu leichtfertig als Melisse gedeutet werden sollte [6]. Der Holzschneider des „Macer“-Frühdruckes entscheidet sich hier für eine Illustration, die viel Spielraum für Interpretationen bietet ( $\mathbf{A b b} \mathbf{2}$ 2).

Nicht abschließend geklärt ist, was Odo in Kapitel 64 mit dem Getreideunkraut 'Lolium' bzw. 'Nigella' meint. Schwarzkümmel oder gar Kümmel sind hier ziemlich sicher auszuschließen. Denkbar ist der Taumel-Lolch (Lolium temulentum), ein Süßgras, das nach Befall durch den Pilz Neotyphodium coenophialum giftig wird. In früheren Zeiten war Getreide und daraus hergestelltes Mehl häufig mit Taumel-Lolch verunreinigt, was zu 


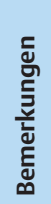

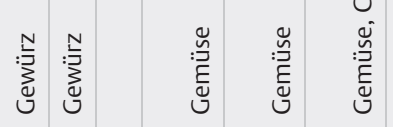

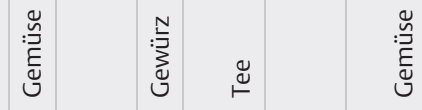

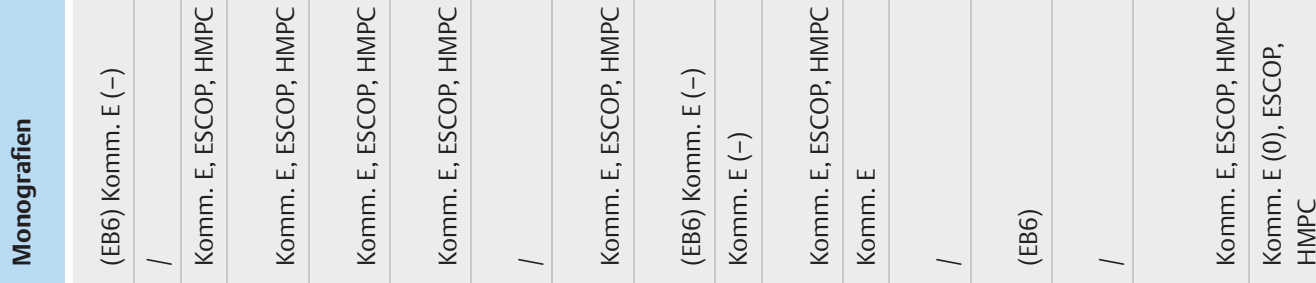

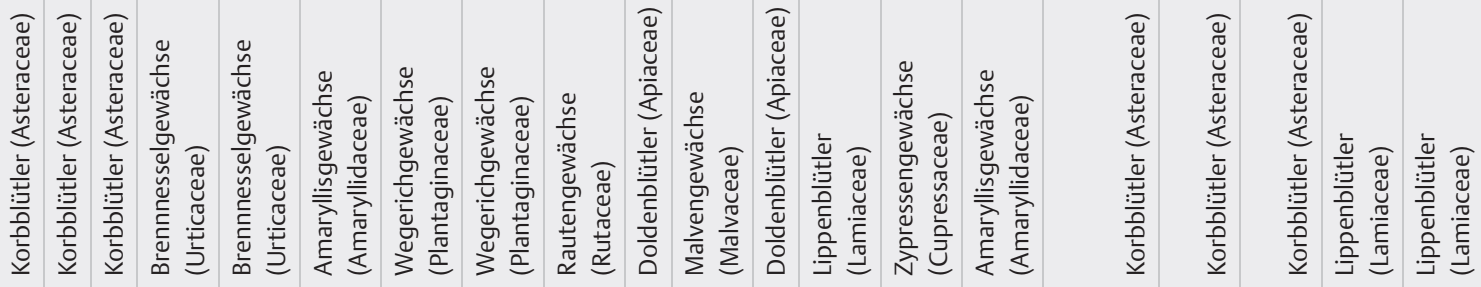

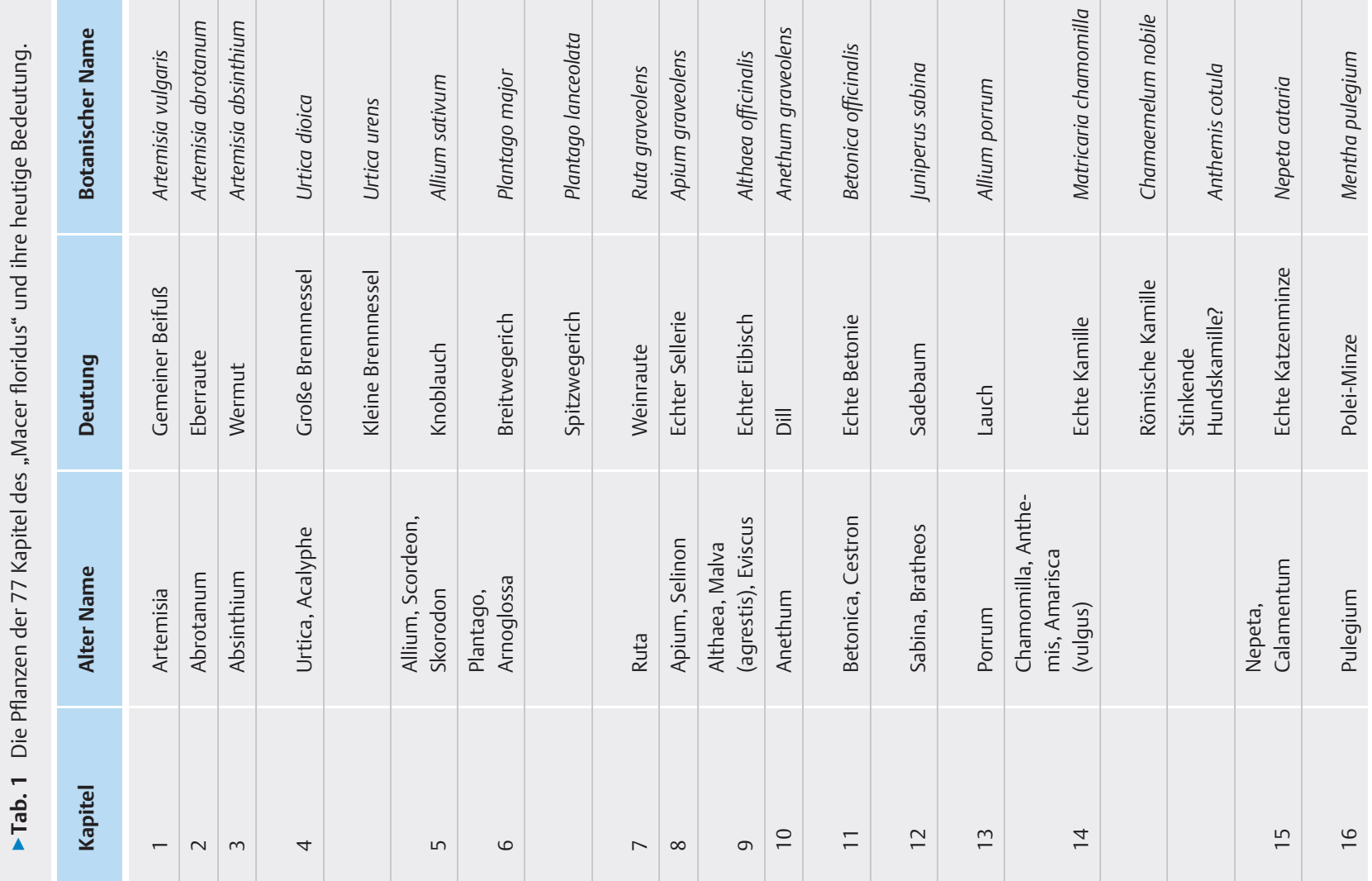




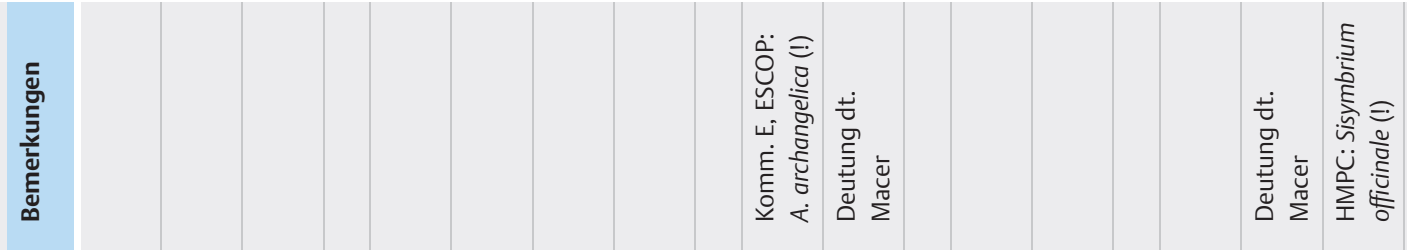

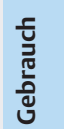

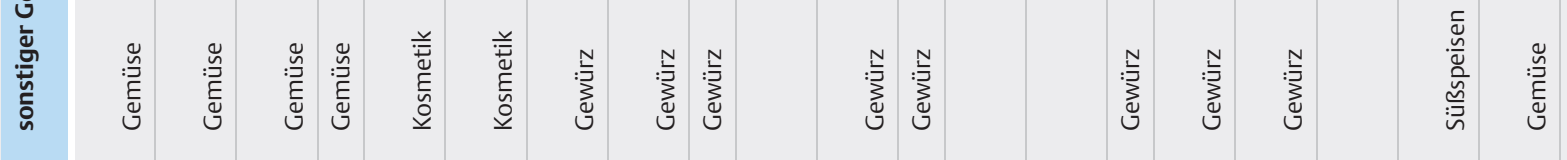

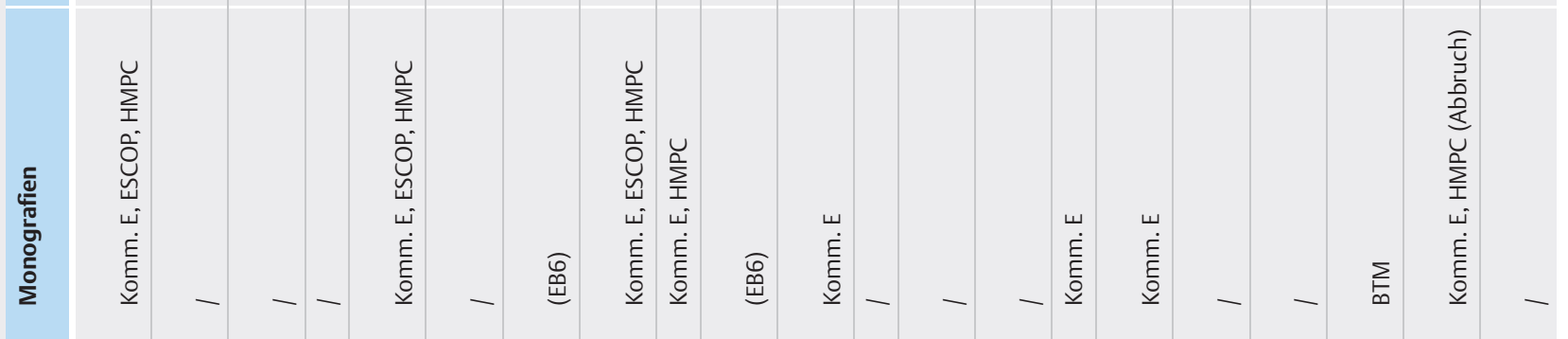

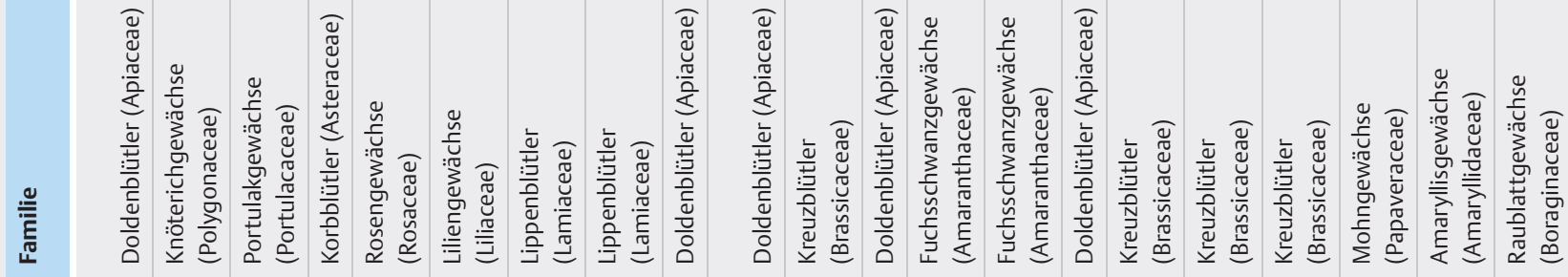

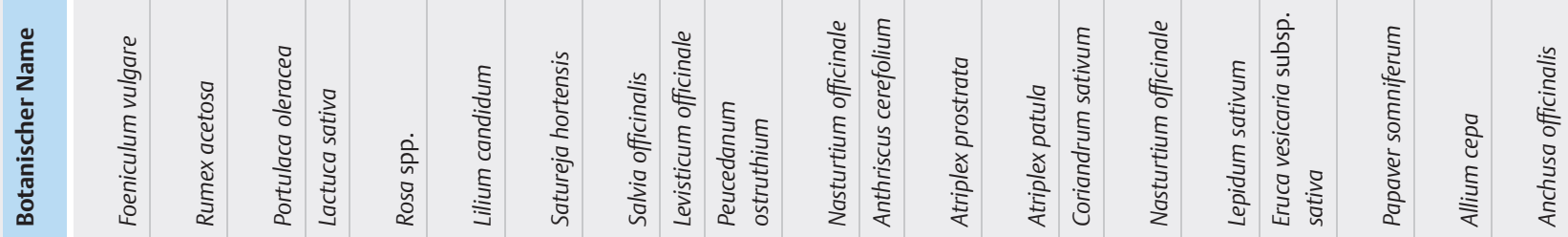

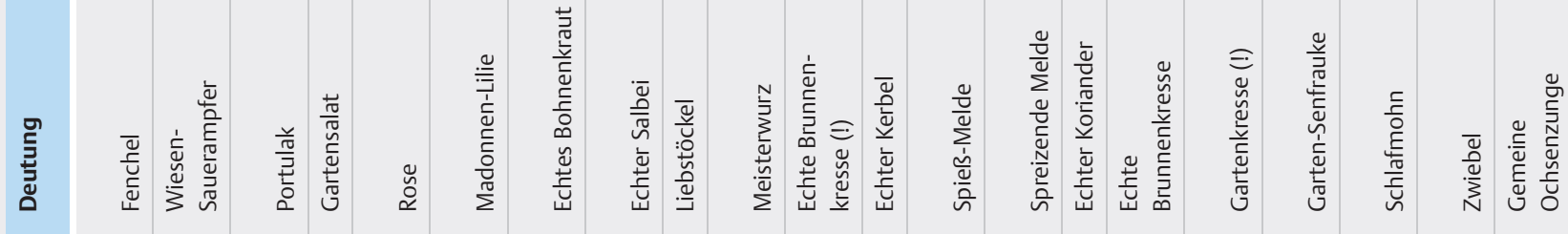

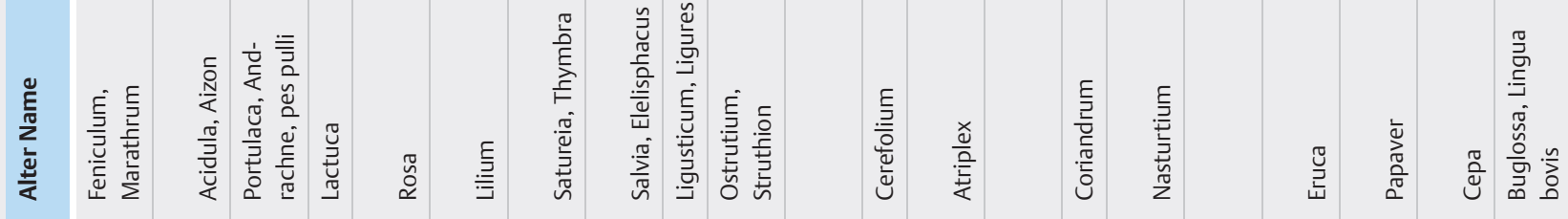

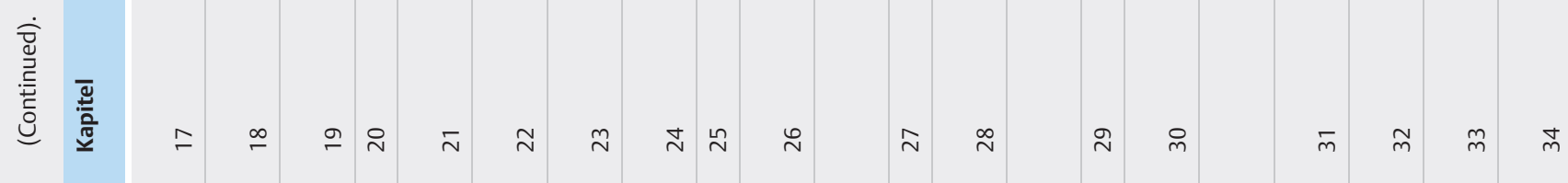



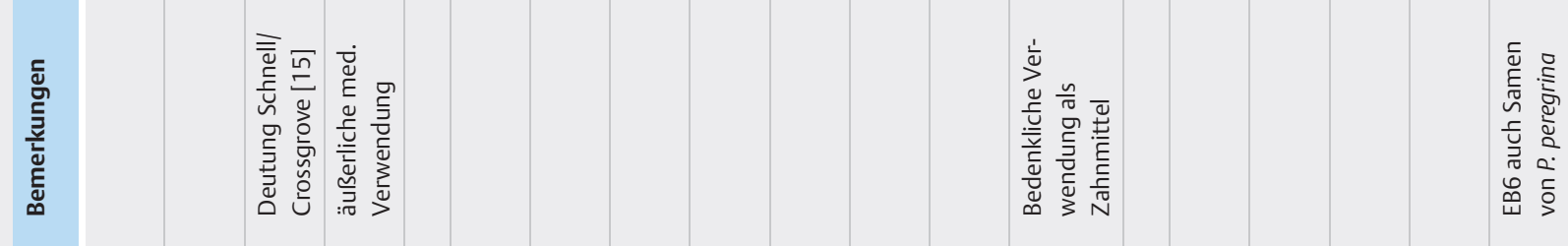

।

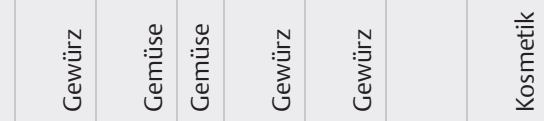

訔 :

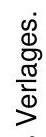

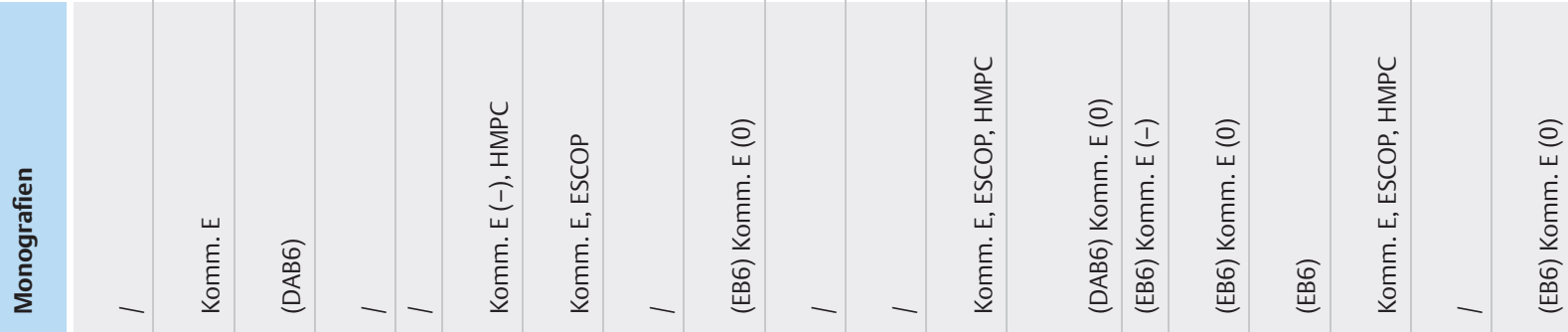

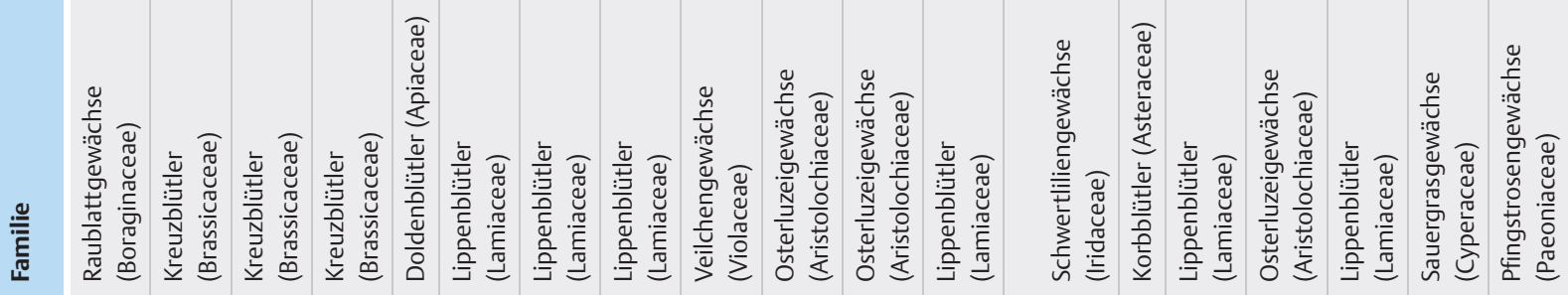

\|\|\|\|\|\|$|\||\||\|| \mid$

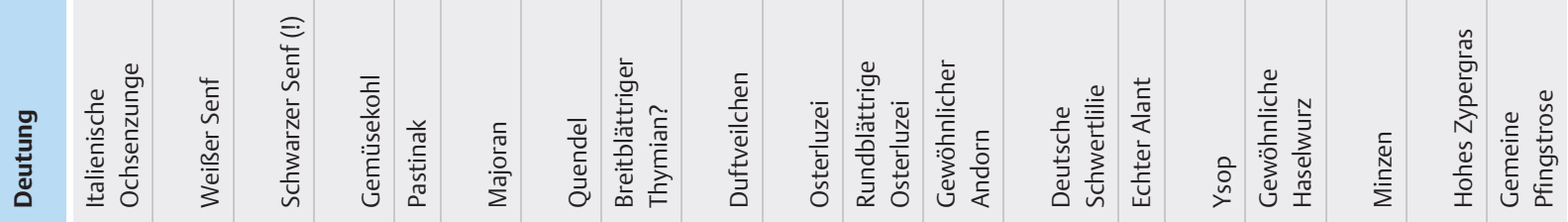

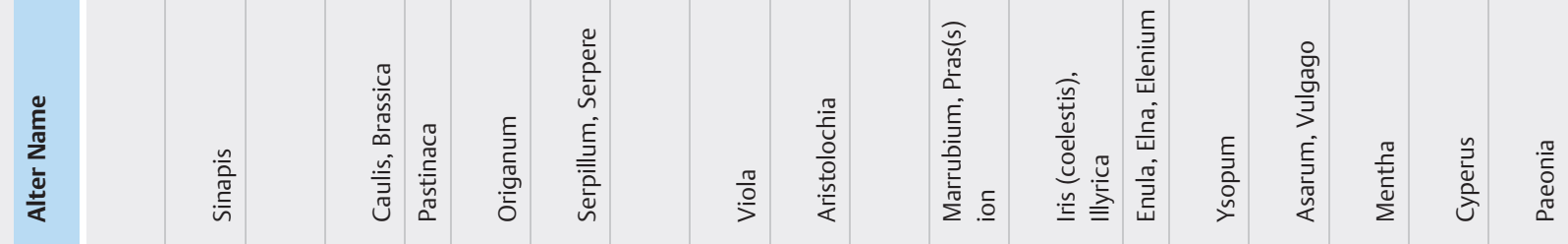

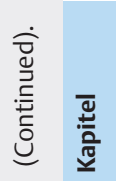

$\stackrel{n}{m}$

$\stackrel{m}{m} \stackrel{\infty}{m} \dot{m}$

ㄱ

ซ $ซ$

ケ 

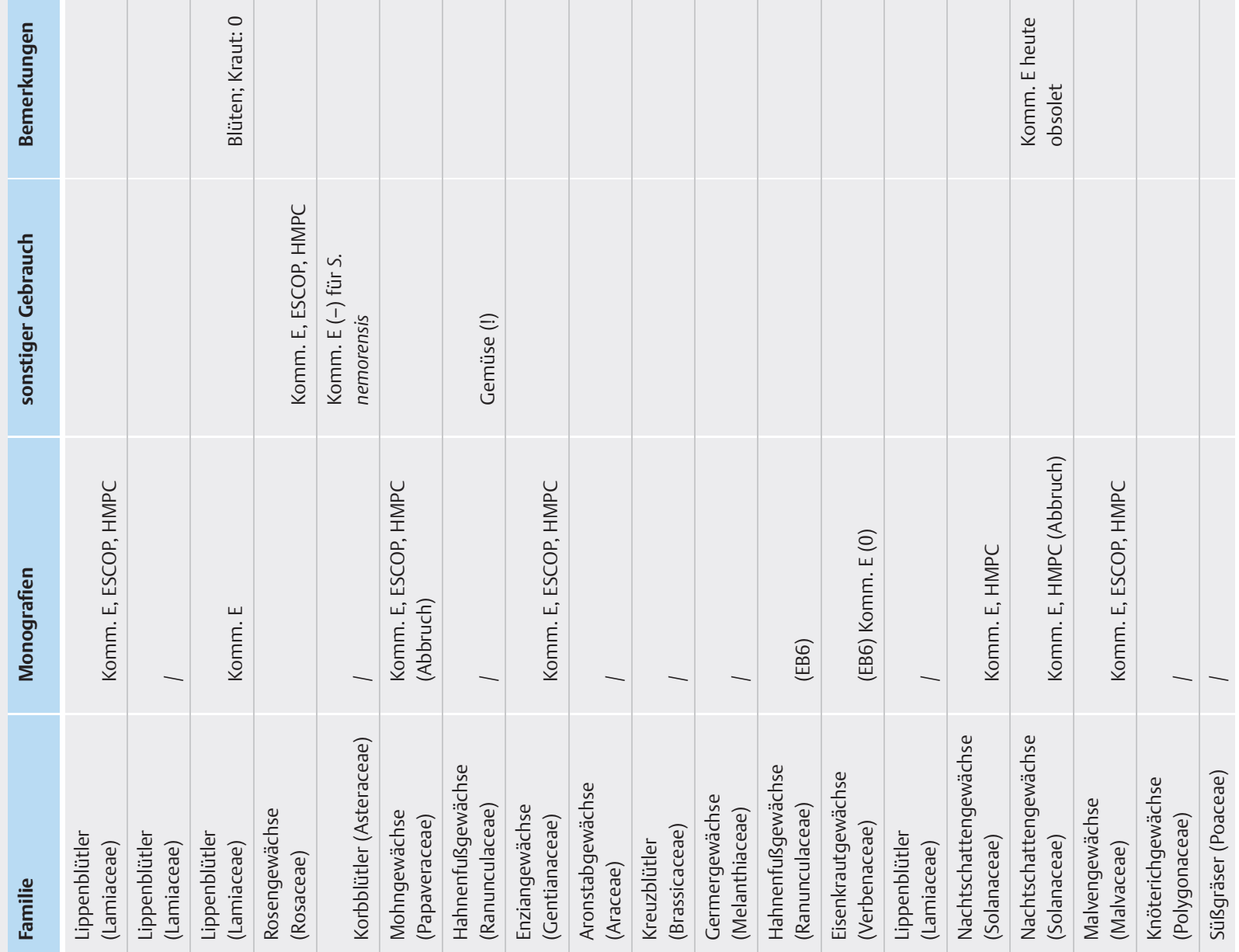

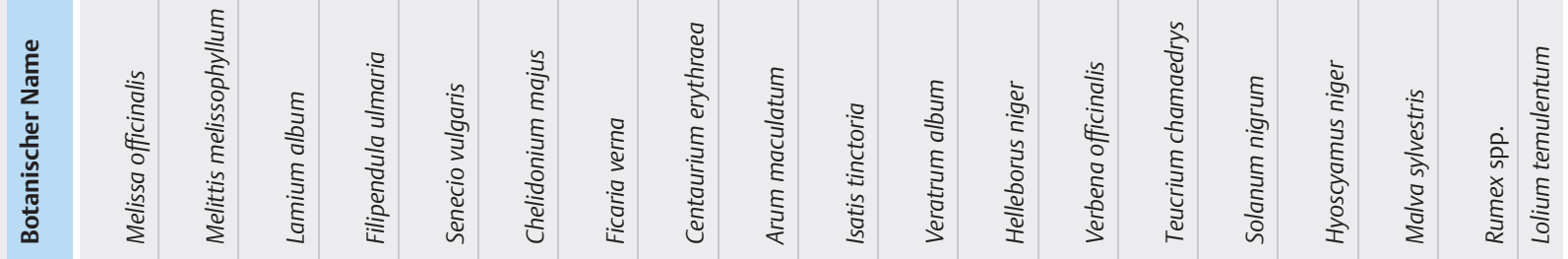

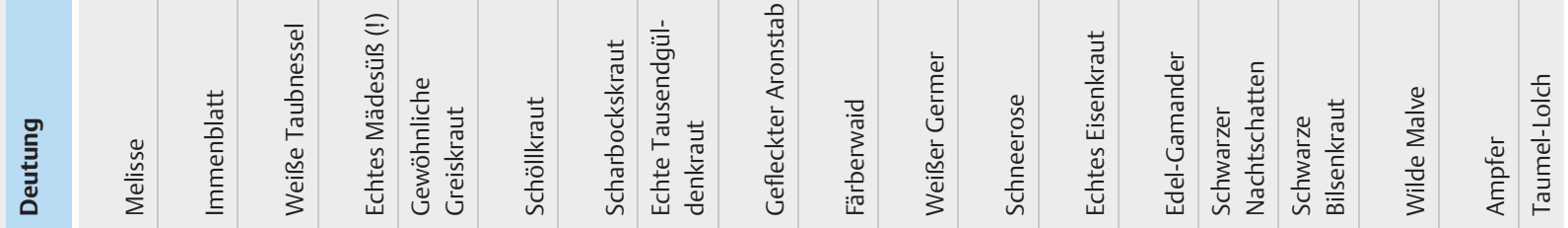

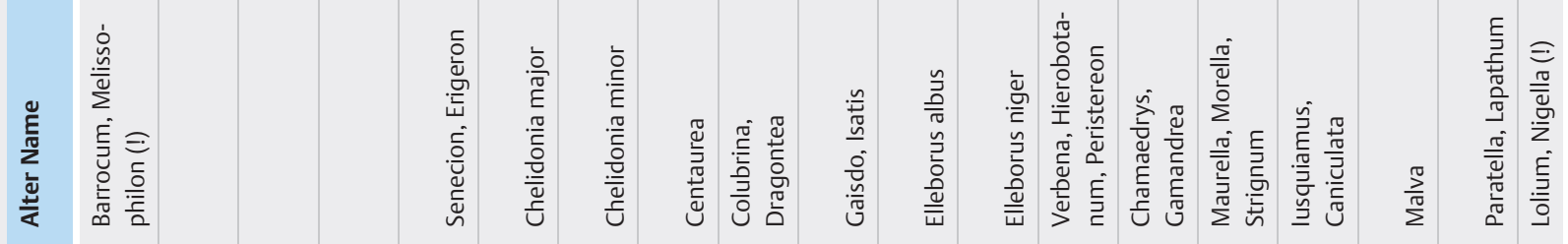

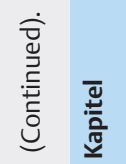

in

in ก

กิ เัก นก

เก

nิ

in

$\begin{array}{llllll}8 & 6 & 6 & 6 & 0\end{array}$ 

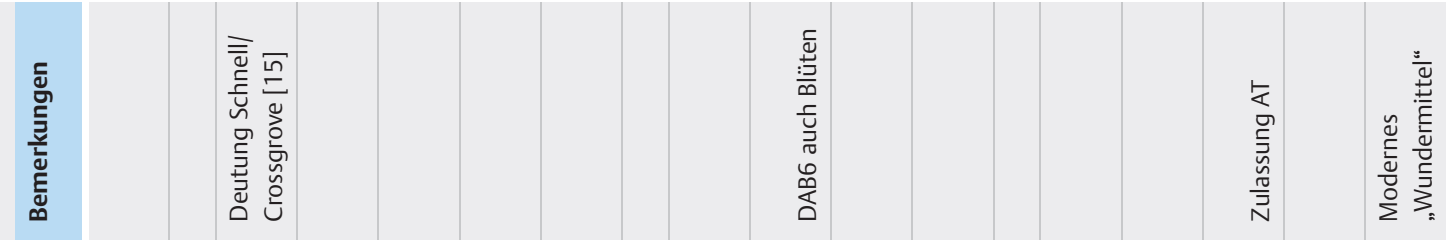

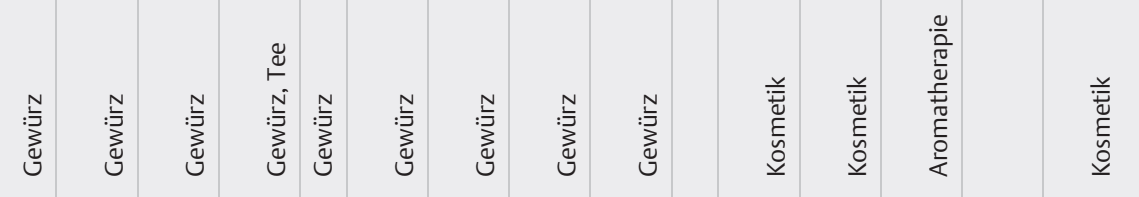

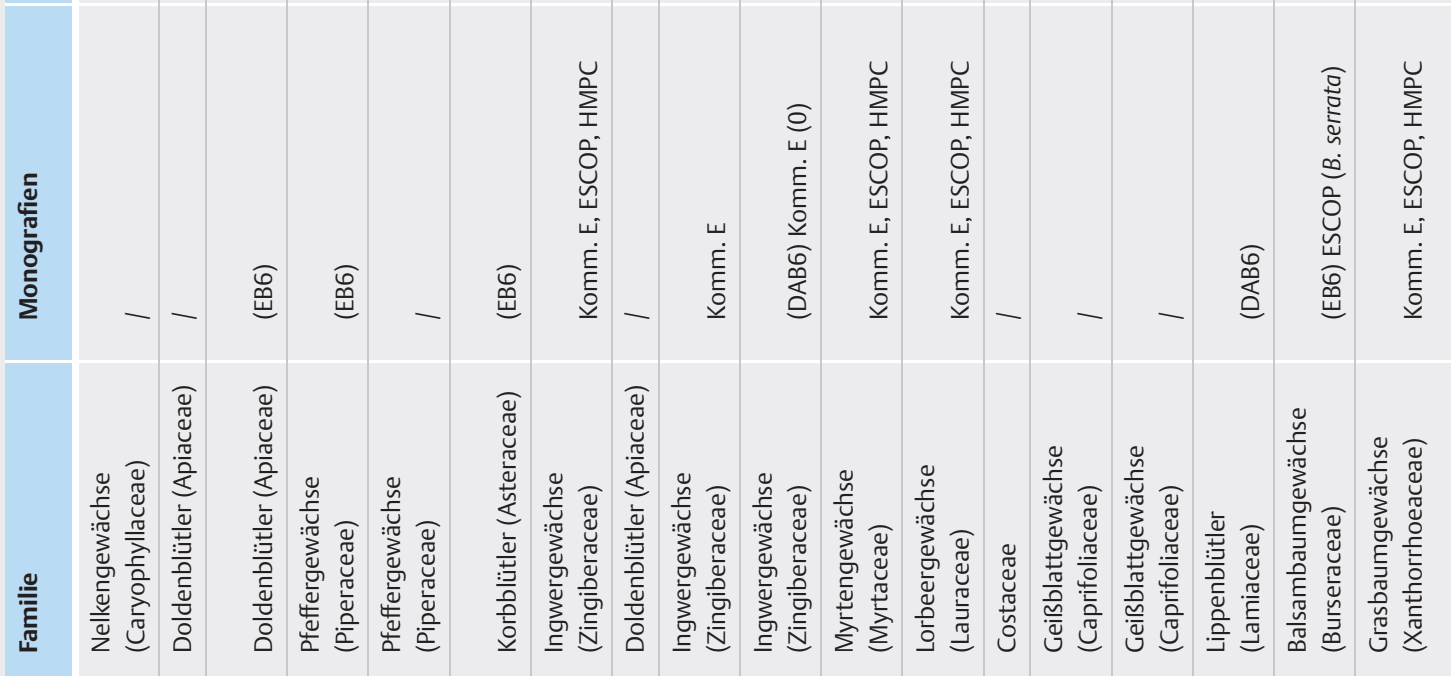

\|\|\|\|\|\|\|\|\|\|$\|$

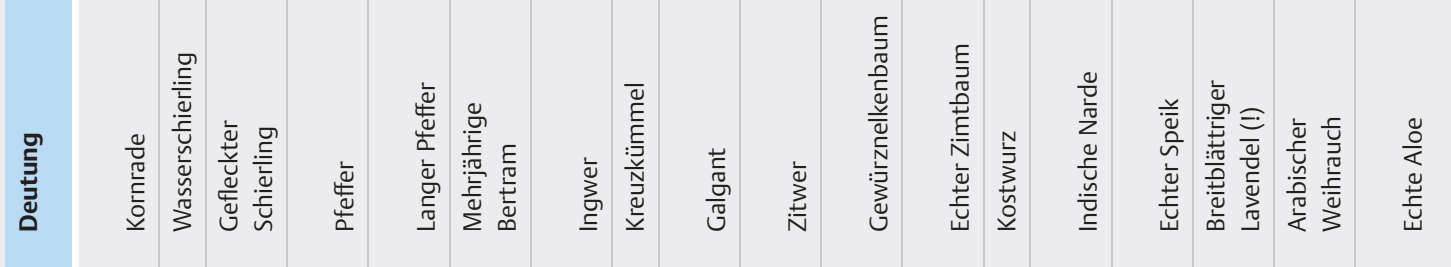

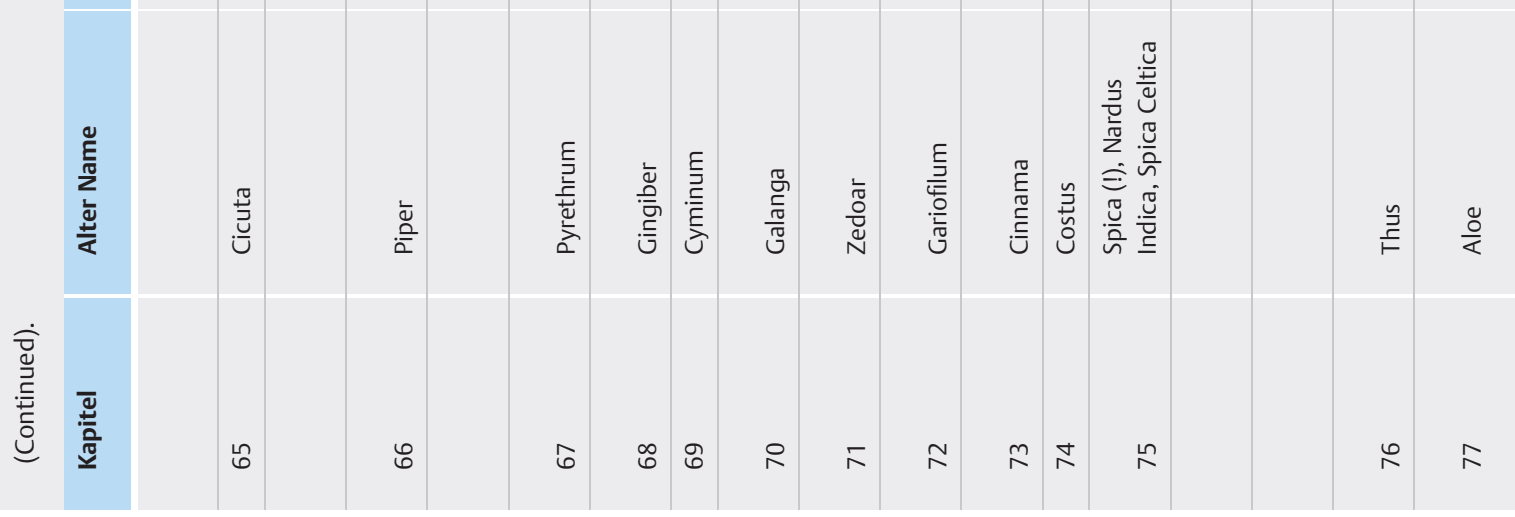




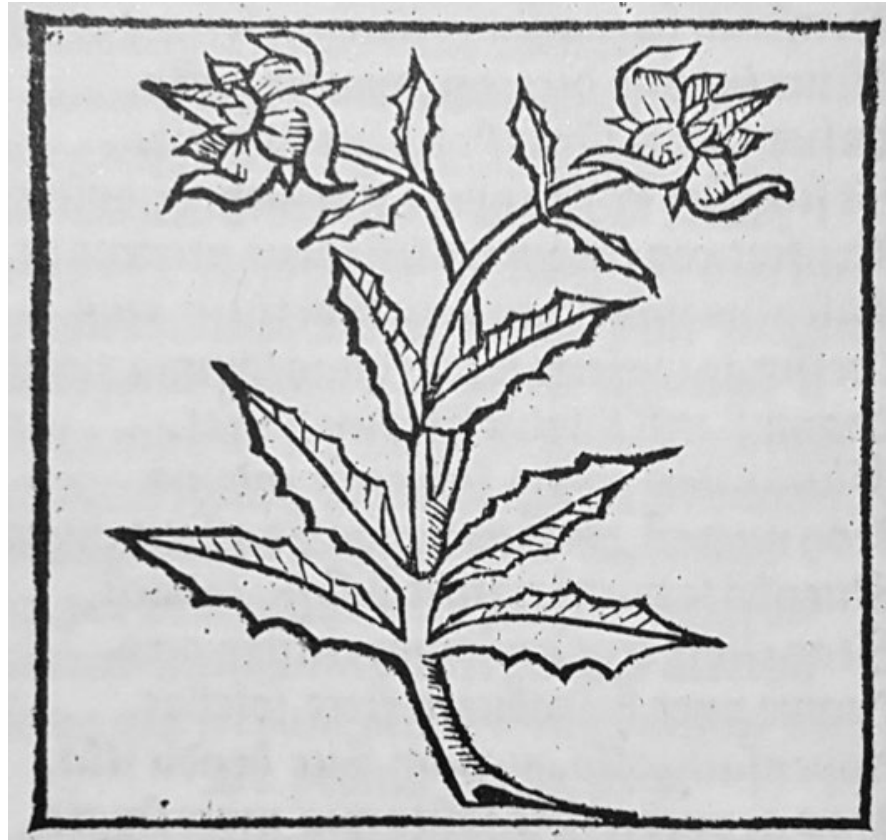

A Abb. 2 „Barrocum“, „Melissophilon“: Die besonders strittige Pflanze aus Kapitel 50.

schwerwiegenden Vergiftungen führen konnte. Ganz so eindeutig ist die Sachlage aber nicht, denn auch die giftige Kornrade (Agrostemma githago) kommt hier in Frage. Darauf deutet nicht zuletzt die deutsche Vulgatfassung hin, in deren 23. Kapitel die Namen 'Nigella' und 'Raten' genannt werden. Diese Unklarheit setzt sich auch in Hildegards Naturkunde (I.12 und I.154) [6] und im "Gart der Gesundheit“ (Kap. 277) [5] fort und bestand evtl. schon bei den mittelalterlichen Schreibern selbst [14]. Im „Macer“-Frühdruck findet sich hier eine Abbildung, die als Süßgras bzw. Getreide zu deuten ist, aber wenig Ähnlichkeit mit dem Taumel-Lolch hat.

Einen Spezialfall bildet das Kapitel 75 zu 'Spica'. Explizit genannt werden hier zwei Pflanzen, die ziemlich sicher als Indische Narde (Nardostachys grandiflora) und Echter Speik (Valeriana celtica) gedeutet werden können. Da aber insbesondere die aus antiken Texten überlieferte Indische Narde in weiten Teilen des Mittelalters kaum verfügbar gewesen sein dürfte, muss hier immer auch der (Speik-)Lavendel als Ersatz in Betracht gezogen werden [13]. Der Frühdruck hat zu diesem Kapitel keine Abbildung.

Bei 'Plantago' im 6. Kapitel ist zu beachten, dass über weite Strecken der Medizingeschichte der Breitwegerich im Vordergrund steht und der Spitzwegerich allenfalls als 'Plantago minor' Erwähnung findet, was sich auch in den mittelalterlichen Abbildungen niederschlägt. In der deutschen Vulgatfassung hat die 'minner wegebreiten' ein eigenes Kapitel nach 'Plantago' (Kap. 6f.).
Bei 'Chelidonia' (Kapitel 52) hingegen muss berücksichtigt werden, dass hier zwei sehr unterschiedliche Pflanzen beschrieben werden. Während das „Große Schöllkraut" (Chelidonium majus) bis in unsere Tage kontrovers diskutiert wird (siehe dazu auch weiter unten), spielt das „Kleine Schöllkraut“ (Scharbockskraut, Ficaria verna) heute medizinisch überhaupt keine Rolle mehr. Im Trivialnamen steckt die frühere Verwendung gegen Skorbut, die jungen Blätter sind vor der Blüte essbar und reich an Vitamin C.

Für drei Pflanzen ohne moderne Monografie gibt es Bewertungen zu verwandten Pflanzen. Die wahrscheinlich in Kapitel 26 unter 'Ostrutium' und 'Struthion' beschriebene Meisterwurz (Peucedanum ostruthium) gilt heute als Allheilmittel in esoterischen Kreisen, wurde jedoch als Arzneipflanze seit dem EB6 von der Echten Engelwurz (Angelica archangelica) verdrängt (mit Monografien von Kommission E und ESCOP). Schon Gerhard Madaus vermerkte 1938: „Die bei den alten Ärzten als Universalmittel gepriesene Meisterwurz hat auch heute kein einheitliches Indikationsgebiet. Im Vordergrund steht die diuretische und diaphoretische Wirkung." Ebenso verwies er auf die Möglichkeit einer Fehldeutung [8]. In der deutschen Vulgatfassung findet sich in Kapitel 43 das Synonym Brunnenkresse für 'Struthion', im folgenden Kapitel dann Gartenkresse für 'Nasturtium' (Kapitel 30 der lateinischen Vorlage).

Die Garten-Senfrauke (Eruca vesicaria subsp. sativa) wird zwar heute nicht mehr medizinisch genutzt, allerdings hat das HMPC eine Monografie zur Weg-Rauke (Sisymbrium officinale) erstellt [1]. Die heute anerkannte Anwendung von Raukenkraut bei Husten findet sich auch in Kapitel 31 zu 'Eruca'. In der deutschen Vulgatfassung ist hier von WeiBem Senf die Rede ('wizen senfe', Kapitel 46), weshalb Bernhard Schnell und William Crossgrove in ihrer Edition den Senf im 26. Kapitel (Kapitel 35 der lateinischen Vorlage) als Brassica nigra deuten [15]. Das EB6 führt unter 'Semen Erucae' den Weißen Senfsamen auf (lat. Semen Sinapis albae), im "Macer floridus“ werden neben dem Samen aber auch das Kraut und die Wurzel beschrieben.

Das Gewöhnliche Greiskraut (Senecio vulgaris) aus Kapitel 51 spielt heute aufgrund der enthaltenen Pyrrolizidinalkaloide keine Rolle mehr. Aus diesem Grund wurde das verwandte Hain-Greiskraut (S. nemorensis) von der Kommission E negativ bewertet.

Interessanterweise wird das Bohnenkraut in der deutschen Vulgatfassung Gartenkohl genannt, während der eigentliche Kohl als Römischer Kohl bezeichnet wird. Gegenüber der lateinischen Fassung sind auch einige aus heutiger Sicht bedeutsame Pflanzen hinzugekommen, darunter Ringelblume, Anis, Süßholz und Meerrettich sowie die ehemals wichtige Alraune. In der Edition kommt die Vulgatfassung so auf 97 Kapitel. 


\section{Befund}

Bei den Gewürzpflanzen fällt auf, dass die Kommission E noch einige positive Monografien erstellt hat (Dill, Koriander, Brunnenkresse, Weißer Senf und Galgant), wo es bislang zu keiner Bearbeitung durch ESCOP oder HMPC gekommen ist. Beim Quendel liegt zusätzlich zur Kommission E auch eine Monografie der ESCOP vor. Im Falle der Zwiebel hat das HMPC das Verfahren abgebrochen. Bei Schöllkraut und vor allem Bilsenkraut sind die positiven Monografien der Kommission E heute aufgrund toxikologischer Bedenken obsolet, auch hier kam es jeweils zum Abbruch des Verfahrens seitens des HMPC. Sollte Odo in Kapitel 50 die Weiße Taubnessel beschrieben haben, so gibt es ebenfalls nur eine Positivmonografie der Kommission E (für die Blütendroge, das Kraut wurde negativ bewertet).

Einzig beim Majoran wurde aus einer negativen Bewertung der Kommission E eine positive des HMPC. Ebenso einmalig ist der Weihrauch, für den es ausschließlich eine ESCOP-Monografie gibt. Odo dürfte allerdings den Arabischen Weihrauch (Boswellia sacra) gemeint haben, während bei uns heute eher der Indische Weihrauch ( $B$. serrata) im Vordergrund steht. Auch das EB6 kennt noch den Arabischen Weihrauch. Zu keiner „Nullmonografie“ der Kommission E gibt es bislang eine positive Bewertung durch ESCOP oder HMPC, wenn man von der möglichen Erwähnung der Römischen Kamille in Kapitel 14 absieht.

Bei zwei Pflanzen gibt es auch heute noch eine medizinische Nutzung ohne Vorliegen einer Monografie: Relevante Zubereitungen des Schlafmohnes fallen unter das BtMG, für die verbreitete äußerliche Anwendung des Kohls bedarf es keiner Präparate und somit auch keiner Monografie.

Unter den Pflanzen, die überhaupt nicht mehr genutzt werden, sind zahlreiche Giftpflanzen wie die Osterluzei, der Schierling oder der Aronstab. Hier sind aus heutiger Sicht allenfalls isolierte Einzelstoffe medizinisch denkbar (und werden teils beforscht), womit wir uns aber außerhalb des eigentlichen Bereiches der Phytotherapie bewegen.

Gerade der Gefleckte Schierling findet jedoch im EB6 noch Erwähnung, entgegen dem „Macer floridus“ sogar als Einnahme (Mittlere Einzelgabe der Droge: $0,1 \mathrm{~g}$, alternativ $0,02 \mathrm{~g}$ eines wässrig-alkoholischen Auszuges). Odo empfiehlt hingegen nur die äußerliche Anwendung (wahrscheinlich meint er allerdings den Wasserschierling), betont mit Verweis auf Sokrates die Giftigkeit und nennt auch einen starken Lauterwein als Gegenmittel. Madaus erwähnt 1938 die Verwendung als Krebsmittel seit dem 18. Jahrhundert und damals aktuell „als beruhigendes und schmerzlinderndes Mittel bei den verschiedensten Krankheiten“ [8].
Weitere Pflanzen, die seit DAB6 und EB6 aus dem Arzneischatz verschwunden sind bzw. keine positive Monografie erhielten, sind Beifuß, Weinraute, Sadebaum, Echtes Bohnenkraut, die bereits erwähnte Meisterwurz, Schwarzer Senf, Duftveilchen, Deutsche Schwertlilie, Echter Alant, Ysop, Gewöhnliche Haselwurz, Gemeine Pfingstrose (das EB6 enthält auch noch die Samen von Paeonia peregrina), Schneerose, Echtes Eisenkraut, Pfeffer sowie die typischen „Hildegard-Pflanzen“ Bertram und Zitwerimmerhin die Pflanzen aus 18 „Macer“-Kapiteln.

Schon damals nicht mehr erwähnt wurden Eberraute, Sellerie, die ehemals so wichtige Echte Betonie, Lauch, Katzenminze, Polei-Minze, Wiesen-Sauerampfer, Portulak, Gartensalat, Madonnen-Lilie, Kerbel, Melde, Ochsenzunge, Kohl, Pastinak, Osterluzei, Zypergras, Greiskraut, Scharbockskraut, Aronstab, Färberwaid, Weißer Germer, Edel-Gamander, Ampfer, Taumel-Lolch bzw. Kornrade, Kreuzkümmel, Kostwurz sowie Indische Narde und Echter Speik - die Pflanzen aus insgesamt 28 Kapiteln.

\section{ABSTRACT}

„Wird erst einmal der Macer gedruckt sein...“ (Once the Macer has been printed...)

Medicinal herbs and their today's significance in the medieval didactic poem "Macer floridus"

"Macer floridus" (also referred to as "De viribus herbarum") is undoubtedly one of the most important works of European herbal medicine. This Latin didactic poem from the 11th century, which comprises 77 chapters with the most important medicinal plants of the time, is largely unknown to the general public today. The explanation for this is not least because it was translated into almost all important European folk languages relatively shortly after its composition and was often incorporated into herbal book compilations in the Middle Ages. While the significance of the work in its entirety faded increasingly during modern times, numerous herbal applications were handed down for centuries.

Interessenkonflikt

Der Autor erklärt, dass kein Interessenkonflikt besteht.

\section{Korrespondenzadresse}

\author{
Tobias Niedenthal \\ Forschergruppe Klostermedizin GmbH \\ Annastraße 26a \\ 97072 Würzburg \\ Deutschland \\ E-Mail: niedenthal@klostermedizin.de
}


Literatur

[1] Braun S, Melzig MF. Wegrauke, Sisymbrium officinale (L.) Scop. Z Phytother 2020; 41: 151-156. doi:10.1055/a-1102-1707

[2] Cabello de la Torre P, ed. Macer floridus. León: Universidad de León; 1990

[3] Fischer H. Mittelalterliche Pflanzenkunde. 2. Nachdruck der Ausgabe München 1929. Hildesheim: Olms; 2001: 269

[4] Haupt J. Ueber das mitteldeutsche Arzneibuch des Meisters Bartholomaeus. Wien: Karl Gerold's Sohn; 1872: 117

[5] Heiss AG, Stika H, De Zorzi N, Jursa M. Nigella in the Mirror of Time: A Brief Attempt to Draw a Genus' Ethnohistorical Portrait. In: von Carnap-Bornheim C et al., Hrsg. Von Sylt bis Kastanas. Festschrift für Helmut Johannes Kroll. (= Offa. Berichte und Mitteilungen zur Urgeschichte, Frühgeschichte und Mittelalterarchäologie. Band 69/70.). Neumünster: Wachholtz Verlag; 2013: 147-169

[6] Hildebrandt R. Physica. Band 3. Berlin, Boston: de Gruyter; 2014: 48; 76; 241f.; $277 f$.

[7] Jansen U. Spuria Macri. Berlin, Boston: de Gruyter; 2013: $1-118$

[8] Madaus G. Lehrbuch der Biologischen Heilmittel. Band 2. Leipzig: Georg Thieme; 1938: 1075ff.; $1614 f f$.

[9] Mayer JG, Goehl K. Höhepunkte der Klostermedizin. Leipzig: Reprint; 2001
[10] Mayer JG, Goehl K. Das Standardwerk der Klostermedizin: der ,Macer floridus'. Z Phytother 2001; 22: 264-269

[11] Mayer JG, Goehl K. Kräuterbuch der Klostermedizin. Leipzig: Reprint; 2003

[12] Mayer JG, Goehl K. Kräuterbuch der Klostermedizin. Darmstadt; Wissenschaftliche Buchgesellschaft; 2013

[13] Niedenthal T, Puchtler E, Uehleke B. „eines so gewaltigen Geruchs, der alle andere übertrifft": Zur Geschichte von Lavendel, der Arzneipflanze des Jahres 2020. Z Phytother 2020; 41: 67-78. doi:10.1055/a-1004-7605

[14] Riha O. Heilsame Schöpfung - Die natürliche Wirkkraft der Dinge. Physica. Beuron: Beuroner Kunstverlag; 2012: 29f.; 135

[15] Schnell B, Crossgrove W. Der deutsche „Macer“: Vulgatfassung. Tübingen: Niemeyer; 2003

Bibliografie

Zeitschrift für Phytotherapie 2020; 41: 233-242

DOI 10.1055/a-1150-9364

ISSN 0722-348X

(C) 2020. Thieme. All rights reserved.

Georg Thieme Verlag KG, Rüdigerstraße 14,

70469 Stuttgart, Germany 